\title{
Activity-Dependent Codevelopment of the Corticospinal System and Target Interneurons in the Cervical Spinal Cord
}

\author{
Samit Chakrabarty, ${ }^{1}$ Brandon Shulman, ${ }^{1}$ and John H. Martin ${ }^{1,2,3,4}$ \\ Departments of ${ }^{1}$ Neuroscience, ${ }^{2}$ Neurological Surgery, and ${ }^{3}$ Psychiatry, Columbia University, and ${ }^{4}$ New York State Psychiatric Institute, New York, \\ New York 10032
}

Corticospinal tract (CST) connections to spinal interneurons are conserved across species. We identified spinal interneuronal populations targeted by the CST in the cervical enlargement of the cat during development. We focused on the periods before and after laminar refinement of the CST terminations, between weeks 5 and 7. We used immunohistochemistry of choline acetyltransferase (ChAT), calbindin, calretinin, and parvalbumin to mark interneurons. We first compared interneuron marker distribution before and after CST refinement. ChAT interneurons increased, while calbindin interneurons decreased during this period. No significant changes were noted in parvalbumin and calretinin. We next used anterograde labeling to determine whether the CST targets different interneuron populations before and after the refinement period. Before refinement, the CST terminated sparsely where calbindin interneurons were located and spared ChAT interneurons. After refinement, the CST no longer terminated in calbindin-expressing areas but did so where ChAT interneurons were located. Remarkably, early CST terminations were dense where ChAT interneurons later increased in numbers. Finally, we determined whether corticospinal system activity was necessary for the ChAT and calbindin changes. We unilaterally inactivated M1 between weeks 5 and 7 by muscimol infusion. Inactivation resulted in a distribution of calbindin and ChAT in spinal gray matter regions where the CST terminates that resembled the immature more than mature pattern. Our results show that the CST plays a crucial role in restructuring spinal motor circuits during development, possibly through trophic support, and provide strong evidence for the importance of connections with key spinal interneuron populations in development of motor control functions.

\section{Introduction}

Corticospinal tract (CST) connections to spinal interneurons are conserved across species. In addition to corticomotoneuronal connections, humans and monkeys have dense CST projections outside the motor pools. Most other mammals have CST projections to interneurons, predominantly or exclusively (Porter and Lemon, 1993; Lemon, 2008). We have taken a developmental approach to identify interneuronal populations in the cervical enlargement targeted by the CST to gain an understanding of the spinal mechanisms underlying development of skilled movement control. Nothing is known about development of CST projections to identified spinal neuron populations, apart from development of direct projections to motor pools in rhesus monkeys (Armand et al., 1997). We used choline acetyltransferase (ChAT) and calcium-binding protein (CBP) immunohistochemistry in kittens to mark spinal interneurons (Huang et al., 2000; Anelli and Heckman, 2005; Gonzalez-Forero et al., 2005).

Received Feb. 12, 2009; revised May 7, 2009; accepted June 7, 2009.

This work was supported by National Institutes of Health Grant NS36835 (J.H.M.). We acknowledge the assistance of XiuLi Wu for histochemistry and Girma Asfaw and Dr. M. Osman for veterinary care. We thank Dr. Claudia Schmauss for discussions about using unbiased stereology and Dr. Holly Moore for use of her microscope and stereology software.

Correspondence should be addressed to Dr. John H. Martin, Department of Neuroscience, Columbia University, 1051 Riverside Drive, New York, NY 10032. E-mail: jm17@columbia.edu.

D01:10.1523/JNEUROSCI.0735-09.2009

Copyright $\odot 2009$ Society for Neuroscience $\quad$ 0270-6474/09/298816-12\$15.00/0
Our study focused on the early postnatal period crucial for CST development. In the cat, the CST projects into the cervical spinal cord during the first postnatal week (PW) and has transient spinal terminations until PW5 (Martin et al., 2009). By PW8, there is an activity-dependent refinement of CST terminations to the mature pattern, with a restricted dorsoventral distribution and loss of most ipsilateral terminations. After refinement, many motor skills begin to emerge in kittens (Villablanca and Olmstead, 1979; Martin and Bateson, 1985) and the M1 motor map begins to develop (Bruce and Tatton, 1980; Chakrabarty and Martin, 2000). The transition to expressing skillful movements in the kitten is probably partly enabled by changes in CST actions on spinal motor circuits.

The goal of this study was to determine the interplay between the developing CST and its target spinal interneurons during the period when the tract refines its termination patterns. Remarkably focal interneuronal projections have been reported for the mature CST (Armand et al., 1985), implying an important, yet unresolved logic. We first determined the localization of the interneuronal markers in the cervical enlargement and whether they showed regional distribution changes from before CST refinement (PW3 and PW4) to when the CST has a mature termination pattern ( $\geq$ PW8). CBP expression (Clowry et al., 2000; Gonzalez-Forero et al., 2005) and ChAT (Phelps et al., 1984) in spinal interneurons are developmentally regulated, and the CST could play a role in this process. To determine whether the CST 
targets or avoids connections with particular interneuron populations, we examined changes in CST projections to ChAT- and CBP-marked interneuron populations before and after this activity-dependent refinement period. Finally, we determined whether CS system activity was necessary for development of spinal interneuron marker expression. This is plausible because perinatal M1 lesion (Gibson et al., 2000) or inactivation (Clowry et al., 2004) leads to reductions in spinal parvalbumin in maturity.

We present evidence that there is a logic to the spinal connections made by the CST early in development before refinement, rather than an absence of spatial specificity. We further show that particular spinal interneuron populations develop within the termination field of the CST during the refinement period and that this depends on CS system activity. This suggests an activitydependent trophic action of the developing CST on its spinal targets. Our results point to a key role for the CST in restructuring spinal motor circuits during development and stress the importance of connections with particular spinal interneuron populations in M1 motor representation and motor control development.

\section{Materials and Methods}

Animals ( $n=11$, normal development and mature; $n=5$, M1 inactivation; $n=1$, saline infusion control) were obtained from an Association for Assessment and Accreditation of Laboratory Animal Care-accredited supplier. Kittens were delivered in litters of four or five along with a lactating mother at postnatal week 2. Columbia University and the New York State Psychiatric Institute Institutional Animal Care and Use Committees approved all experimental procedures.

General surgical procedures. For all surgical procedures, animals were administered atropine $(0.04 \mathrm{mg} / \mathrm{kg}$, i.m.). A mixture of acepromazine $(0.03 \mathrm{mg} / \mathrm{kg}$, i.m.) and ketamine hydrochloride $(32 \mathrm{mg} / \mathrm{kg}$, i.m.) was given to induce anesthesia. Cats were intubated after anesthesia was induced and maintained in an areflexive condition during surgery using $1-2 \%$ isoflurane. Animals were given a broad-spectrum antibiotic at the time of surgery (cefazolin; $25 \mathrm{mg} / \mathrm{kg}$, i.m.) and an analgesic after surgery (buprenorphine; $0.03 \mathrm{mg} / \mathrm{kg}$, i.m.). Animals resumed nursing after recovery from anesthesia, and were given supplemental milk [feline milk replacement (KMR); PetAg] as needed to ensure adequate weight gain.

M1 activity blockade. To block neuronal activity, the $\mathrm{GABA}_{\mathrm{A}}$ agonist muscimol (10 $\mathrm{mm}$ in sterile saline; Sigma) was continuously infused using an osmotic minipump (model 2002; Alzet) $(0.5 \mu \mathrm{l} / \mathrm{h})$ into the center of the motor cortex forelimb representation, located primarily in the lateral sigmoid gyrus (Chakrabarty and Martin, 2000), as in our previous studies (Martin et al., 1999; Friel and Martin, 2005). A 28 gauge hypodermic needle cannula (Alzet), beveled at the tip, was connected with vinyl tubing (size 4; Scientific Commodities) to the pump. The cannula was inserted below the pial surface. The cannula was fixed to the skull with screws and dental acrylic cement. Neuronal activity was blocked in left M1 from PW5 to PW7. The osmotic pump delivered muscimol for 2 weeks. Animals either were killed at the end of the infusion period, or the cannula and pump were removed and the animals were allowed to survive for an additional 4 weeks. One animal received saline infusion between PW5 and PW7.

A variety of histochemical markers were used to assess the spatial effect of muscimol. Using the metabolic marker cytochrome oxidase as an indication of cortical function, muscimol infusion produces a maximal reduction within a $2.5-3 \mathrm{~mm}$ radial patch of cortex at the infusion site and a lesser reduction for an additional 4-5 mm (Martin et al., 1999). This suggests that the muscimol infusion produced maximal inhibition within the center of the M1 forelimb representation and reduced inhibition over a larger territory that extended into area 6 rostrally and somatic sensory cortex caudally. A similar distribution of reduced staining of the calcium-binding protein parvalbumin was produced by muscimol infusion (Friel et al., 2007). We showed previously that the infusion did not produce a lesion by comparing cell body density (Martin et al., 2000) and the distribution of neurofilament-F (SMI-32) immunoreactivity (Friel et al., 2007). We also found that the medullary pyramid cross-sectional areas on each side and SMI-32 staining in the lateral spinal column on each side were not different (Martin et al., 1999; Friel and Martin, 2005).

Corticospinal axon tracing. To trace the CST, we pressure injected (Picopump; World Precision Instruments) the anterograde tracers biotinylated dextran amine (BDA; Invitrogen; 10\% in PBS) into the forelimb area of M1 of one hemisphere and Lucifer yellow-dextran amine (LYDA; Invitrogen, 1\% in PBS) into either M1 of the other hemisphere or another portion of M1 forelimb representation in the same hemisphere. For most of the normally developing animals, we used a standard pattern of tracer injection into the lateral sigmoid gyrus ( 3 injections; $300 \mathrm{nl}$ each; $1.5 \mathrm{~mm}$ below the pial surface; separated by $1.0-1.5 \mathrm{~mm}$ ). In selected animals (see Results), injections of one or the other tracer were made within different M1 subregions. For PW3 and PW4 animals, injections were made 2 weeks before killing each animal, and for older animals, 4 weeks before killing the animals. For determining the CST projection from inactivated M1, tracer injections were made 3-7 d after cessation of the infusion (i.e., PW8). The three injections were positioned rostral, lateral, and caudal to the muscimol infusion site, as in our previous studies (Friel and Martin, 2005, 2007). Importantly, this ensured that we were examining the previously silenced CST projection. We determined that the infusion had the same effect on CST development in these animals as in our other studies. However, we do not report these data, as they were identical to those previously published.

Histology and tracer histochemistry. Cats were deeply anesthetized (sodium pentobarbital; $30 \mathrm{mg} / \mathrm{kg}$, i.v.) and perfused transcardially with warm saline, followed by a solution of $4 \%$ paraformaldehyde, $\mathrm{pH}$ 7.4. Heparin was injected (200-500 U, i.v.) at the onset of perfusion. For perfusion, a peristaltic pump was used at a predetermined flow rate that depended on the animal's weight. The total perfusion time was 20-30 min. The brain and spinal cord were removed, postfixed in the same fixative for $2-3 \mathrm{~h}$, and then transferred to $20 \%$ sucrose in $0.1 \mathrm{M}$ phosphate buffer overnight at $4^{\circ} \mathrm{C}$. Frozen transverse sections $(40 \mu \mathrm{m})$ through the cervical spinal cord (C6-C8) were cut and processed for BDA histochemistry and LY-DA immunohistochemistry to determine the distribution of labeled CS terminals. Cervical spinal sections were also processed for ChAT and calcium-binding protein (CBP) immunocytochemistry (see below). Parasagittal sections through the cortex were cut and alternate sections were processed for BDA or LY-DA, to determine the location of tracer injection sites, and Nissl stained for assessing cortical cytoarchitecture.

For visualization of BDA, sections were incubated in PBS containing the avidin-biotin complex reagent as prescribed by the manufacturer (ABC kit; Vector Laboratories) and $0.2 \%$ Triton for $2 \mathrm{~h}$ at room temperature (RT). After rinsing, sections were incubated with the chromogen diaminobenzidine (DAB; Sigma) for 6-30 min. To examine the relation between $\mathrm{BDA}$ and interneuron markers, we used a fluorescent fluorophore. The sections were incubated with conjugated ExtrAvidin FITC (1:200, Sigma) or ExtrAvidin Cy3 (1:500-1:800, Sigma) overnight at $4^{\circ} \mathrm{C}$. After rinsing, sections were mounted on gelatin-coated slides, air dried overnight, and coverslipped. To visualize axons labeled with LY-DA, sections were incubated at $4^{\circ} \mathrm{C}$ overnight in PBS containing rabbit antiLY-DA antibody (1:1000; Invitrogen) in blocking buffer (3\% donkey serum in $1 \times \mathrm{PBS}$ with $0.2 \%$ Tween, $\mathrm{pH} 7.4$ ). After rinsing, sections were incubated for $2 \mathrm{~h}$ at RT in blocking buffer containing $0.2 \%$ anti-rabbit secondary antibody conjugated to peroxidase (1:200; $\mathrm{pH} 7.4)$. After rinsing, sections were incubated with the chromogen DAB for 5-30 min. As with BDA, we also used fluorescence to mark LY-DA. We either used a secondary antibody conjugated to $\mathrm{Cy} 3$ (1:800; incubated at RT; $1 \mathrm{~h}$; donkey anti-rabbit, -goat, or -mouse), or to FITC (1:500; RT; 2 h; donkey anti-rabbit, -goat, or -mouse).

Calcium-binding protein and ChAT immunohistochemistry. We used immunohistochemistry to visualize the distribution of cells containing choline acetyltransferase (ChAT), calbindin (CB), parvalbumin (PV), and calretinin (CR), as others have used in cat, rat, and mouse spinal cord (Clowry et al., 2000; Gibson et al., 2000; Clowry et al., 2004; Anelli and Heckman, 2005). ChAT-positive interneurons are implicated in locomotor control (Huang et al., 2000). CR is thought to mark excitatory interneurons predominantly, and $\mathrm{CB}$ and $\mathrm{PV}$, different classes of inhibitory 
interneurons (Kubota and Jones, 1993; Albuquerque et al., 1999; Schwaller et al., 2002).

Each primary antibody staining was visualized by both fluorescent and peroxidase conjugated secondary antibodies. Each antibody was tested for efficacy for both the fluorescent and DAB methods using a range of primary antibody concentrations. While is was not possible to coincubate all tissues from cats of different ages, we routinely coincubated tissue from selected pairs of animals to verify that age-related differences were not due to subtle differences in immunohistochemical reactions. For fluorescence labeling, we used both FITC- (AlexaFluor 488; Invitrogen) and Cy3- (Jackson ImmunoResearch Laboratories) conjugated secondary antibodies. The final concentration used produced optimal labeling at all ages examined. No differences were noted between the two methods. We report only results based on the fluorescence method.

The following antibodies (with epitopes, according to the manufacturers and published data) were used, at the concentrations and incubation times indicated: (1) rabbit polyclonal anti-CB D-28K (Millipore; AB1778, CB; SwissProt: P05937; 261 aa; single band on Western blot, 28 $\mathrm{kDa} ; 1: 500 ; 4^{\circ} \mathrm{C}, 2 \mathrm{~d}$ ); (2) mouse monoclonal anti-PV [Swant; PV235, purified from carp muscle; two-dimensional immunoblot stains the ${ }^{45} \mathrm{Ca}^{2+}$-binding spot of PV (single spot staining) (Celio et al., 1988); $1: 5000 ; 4^{\circ} \mathrm{C}$, overnight], (3) mouse monoclonal anti-CR (Millipore; MAB1568, clone 6B8.2; SwissProt: P22676; 271 aa; single band on Western blot, $31 \mathrm{kDa} ; 1: 1000 ; 4^{\circ} \mathrm{C}$, overnight), and (4) goat polyclonal antiChAT (Millipore; AB144P; SwissProt: P28329; 748 aa; single band on Western blot, $68-70 \mathrm{kDa}$; 1:100; $4^{\circ} \mathrm{C}$, overnight).

For each primary antibody, free-floating sections were incubated at RT in PBS containing the primary antibody in blocking buffer (3\% donkey serum in $1 \times \mathrm{PBS}$ with $0.2 \%$ Tween, $\mathrm{pH} 7.4$ ). After rinsing, sections were incubated at RT in blocking buffer containing secondary antibody conjugated to Cy3 (1:800; RT; $1 \mathrm{~h}$; donkey anti-rabbit, -goat, or -mouse), to FITC (1:500; RT; 2 h; donkey anti-rabbit, -goat, or -mouse), or to peroxidase (1:200; RT; 2 h), at $\mathrm{pH}$ 7.4. For fluorescence labeling, sections were mounted on gelatin-coated slides, air dried, and coverslipped with Vectashield (Vector Laboratories). For peroxidase staining, sections were dehydrated before coverslipping.

We conducted double-label immunofluorescence for the following pairs of markers: ChAT-CR; ChAT-PV; ChAT-CB; ChAT-LY-DA; PVCB; PV-CR; PV-LY-DA; CB-CR; and CB-LY-DA. We could not do CR-PV because both primary antibodies were generated in the mouse. For double labeling, we coincubated the primary antibodies, using the same protocols as above. Then, we incubated in secondary antibody solutions, also as above.

Topography of CS terminations. We used a quantitative method for determining the topographic distribution of label within the gray matter in the cervical enlargement (Brus-Ramer et al., 2007; Friel and Martin, 2007). BDA- and LY-DA-labeled axons were traced from transverse spinal sections at $200 \times$ magnification using Neurolucida (Microbrightfield), by moving the cursor along the length of the labeled axons. CS terminations are defined as axon label within the gray matter in spinal sections. Varicosities are defined as punctate axonal swellings with a diameter more than three times the diameter of the nonvaricose axon. Varicosities along axons were marked. CS axon varicosities are putative presynaptic sites (i.e., boutons) because they colocalize synaptophysin (Meng et al., 2004). For each cat, all axon label (BDA or LY-DA) was traced on each section. The number of sections traced was determined by the amount of label on each section. Sections were added until the total length of axon that had been traced reached $\sim 25,000 \mu \mathrm{m}$.

Tracings of axon label and varicosities were separately exported and quantified using a suite of programs written in Matlab (MathWorks). Briefly, a program divided the gray matter into $30 \times 30 \mu \mathrm{m}$ square regions of interest (ROIs). For each ROI, we computed the mean density of traced axons or marked varicosities. All axonal tracings and varicosities were one pixel wide. This corresponds to the amount of label (i.e., total number of pixels) in the ROI divided by the area of the ROI. A matrix of mean axon or varicosity density was generated in Matlab that preserved the mediolateral and dorsoventral dimensions of the distribution of label in the gray matter. At the same time, the borders of the gray matter were traced to obtain a precise correspondence between labeling and spinal cord structure. We quantified the regional axon and varicosity density to generate maps of regional distribution of axonal label distribution and to quantify the amounts of label in different spinal laminae (Rexed, 1954). Density is represented according to a color scale, from the lowest density (blue) to the highest (red). Background is very dark blue. Regional distribution maps were generated for individual animals and averaged maps for all animals within a particular age or treatment group. For the averages, we aligned the data from different animals according to the point of intersection between the gray matter above the central canal and the dorsal median septum. We also generated composite regional distribution maps (see Fig. 5) for immature animals ( $\leq$ PW8) and mature animals ( $\geq$ PW8). These were constructed by averaging CST tracings following tracer injection into rostral $(n=2 ; \leq \mathrm{PW} 8 ; n=2 ; \geq \mathrm{PW} 8)$ and caudal $(n=2$; $\leq$ PW8; $n=2$; $\geq$ PW8) M1, reanalyzed from animals of previously published studies (Li and Martin, 2000, 2001). The composite maps are used as a proxy for making multiple extensive injections in individual animals.

Image acquisition. Bright-field and epifluorescence images were acquired on an Olympus BX-60 microscope using $10 \times$ [numerical aperture (NA) 0.3], 20× (NA 0.5) and $40 \times$ (NA 0.75) objectives and a Microfire-CCD camera (Optronics) mounted on the microscope. Unbiased cell counting (see below) was done using a Zeiss Axioplan2 with a Microfire-CCD camera. For generating composites to create regional distribution maps to visualize the locations of cells containing the CBPs (e.g., Fig. 1, left), a Zeiss inverted Axioscope 200M was used to scan the gray matter of the spinal section at $100 \times$ (NA 0.5 objective) using z-series image capture and deconvolution software (Volocity; Improvision, PerkinElmer). After deconvolution (8 runs using a measured point spread function for the lens used), images of each $\mathrm{Z}$ plane were then merged. Using Mosaic J, a composite for each section was then made. For epifluorescence, a 460-500 $\lambda$ excitation filter and a 510-560 $\lambda$ emission filter was used for FITC; and a 535-550 $\lambda$ excitation filter and a 610-675 $\lambda$ emission filter was used for Cy3. To adjust contrast and brightness, Adobe Photoshop (Adobe Systems) was used. When comparing images, all capture and adjustment parameters were kept identical.

Regional distribution maps of the CBPs. The composite deconvolution images were imported into Neurolucida as Tiff files, generated using ImageJ. The cell locations were then marked at $200 \times$ in Neurolucida. For marking interneurons, we were agnostic to neuron size, but took into account standard neuronal morphology, requiring that a marked profile had one or more processes emanating from the cell body. Whereas overall marking was done at $200 \times$, we occasionally went to higher magnification to determine whether or not to mark a cell. The locations of the cell bodies and the gray matter borders were exported to a suite of programs written in MATLAB (see methods above for the CST terminations) that allows us to generate the regional distribution map (i.e., heat map) for the particular CBP for the section scanned. Each map is scaled to the individual animal's data. We aimed to achieve the full dynamic range of the color scale, without distortion due to nonlinear mapping inherent in false-color imaging. When there was a single dense cell cluster, this resulted in saturation of a few pixels within that cluster. It was often the case, however, that there were multiple clusters with a similar cell density. In these cases, multiple sites of saturation emerged concurrently. For each animal at least 3-5 such sections were scanned and converted and averaged. A contour finding routine was written in Matlab and applied to the regional distribution map, where the density of CBP and ChAT cells was equal or $>60 \%$ of maximal density. This closely approximates the regions defined by yellow through saturated red. For the purposes of comparing across animals, the gray matter border and contours for individual markers were grouped using a graphics program (FreeHand, Macromedia/Adobe) and the size of the gray matter, and associated contours, was normalized to a standard dorsoventral length. Figures 4 and 5 show all data from all animals. It can be seen in the figure that the distributions of cell clusters were not biased by subtle differences in gray matter widths. For comparing cell groups and CST labeling in individual animals (see Fig. 6), contours were transferred directly to the regional distribution maps, without any size adjustments, being careful to ensure precise alignment of the two images.

Cell counts. To obtain quantitative estimates of the density of marked 


\section{A. ChAT-PW8}

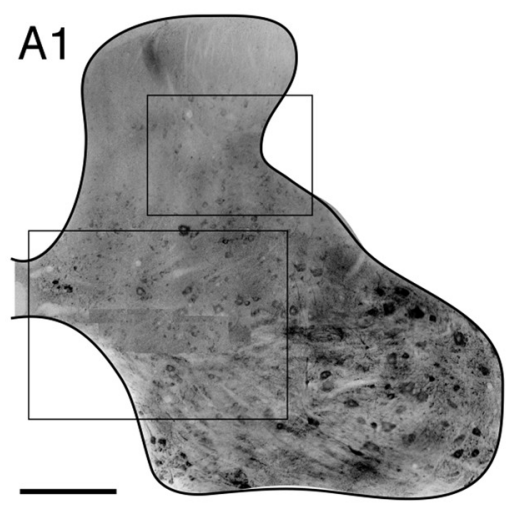

\section{B. CB-PW3}

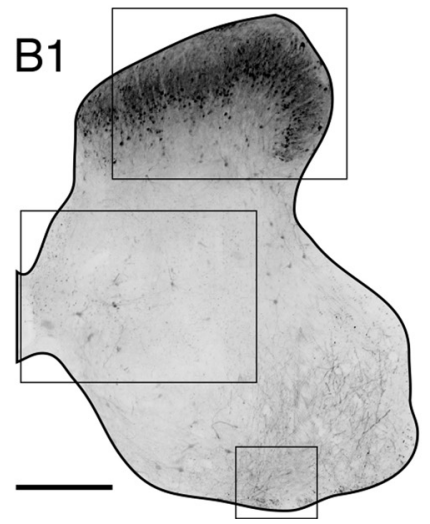

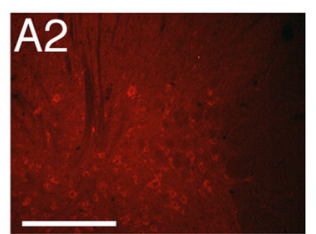

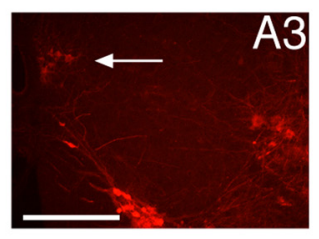

A3

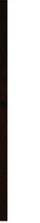
西

C. CR-PW3
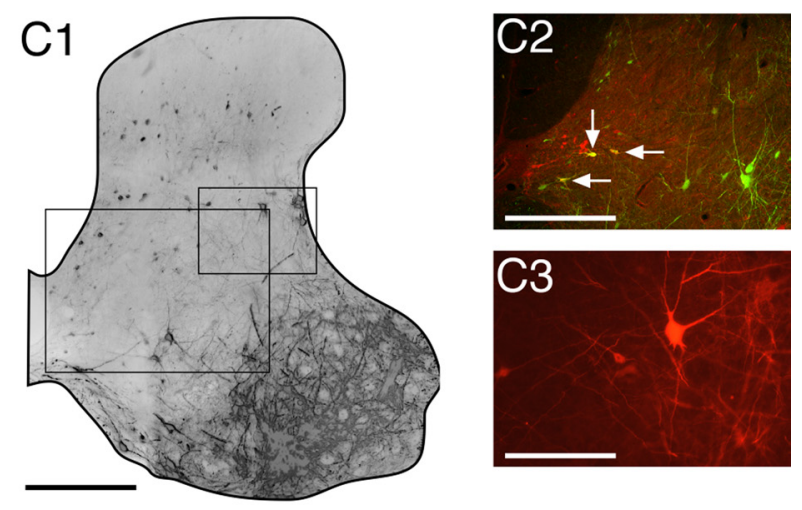

D. PV-PW3
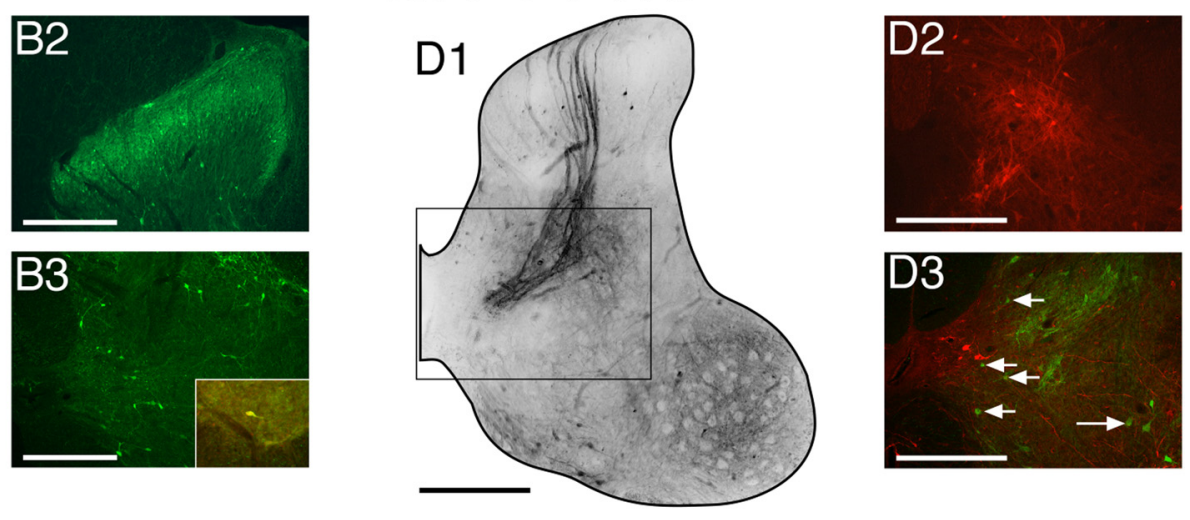

Figure 1. Typical expression pattern of interneuron markers (ChAT, CB, CR, PV) in the cervical enlargement (C6-C8) of the spinal cord. Left, Montages obtained by deconvolution confocal microscopy. Right, Single- or double-labeled epifluorescence micrographs, at higher magnification. Boxes in left panel figures show the approximate locations of micrographs in the right panels. $A$, ChAT. $\boldsymbol{A} \mathbf{1}$ is a montage from a PW8 animal. $\boldsymbol{A} \mathbf{2}$ is spinal section stained for ChAT (secondary antibody conjugated to Cy3; red) showing interneurons throughout laminae 5-7. A3 shows ChAT interneurons that are consistently present medially in lamina 7. The arrow points to $C h A T$ interneurons close to the central canal. $\boldsymbol{B}, \mathrm{CB} . \mathbf{B} 1$ is a montage from a PW3 animal. $\boldsymbol{B} 2$ shows $\mathrm{CB}$-positive interneurons (secondary antibody conjugated to FITC, green) in the dorsal horn. $\mathbf{B} 3$ shows (B staining in the intermediate zone and ventral horn. $\boldsymbol{B} 3$ inset shows a double-labeled interneuron, with CB staining (FITC) and PV staining (Cy3), in the ventral lamina 7 (lower small box, B1). C, CR. C1 shows a montage from a PW3 animal. C2 shows (R-positive interneurons (FITC) clustered medially in lamina 7. The arrows point to CR interneurons that also stained for ChAT (Cy3; double labeled, yellow). C3. CR interneurons (Cy 3; red) located laterally in the deep dorsal horn and intermediate zone. Note that the dense CR motor pool staining in the deconvolution image reflects dense dendritic (or axonal) processes. D, PV. D1 is a montage from a PW3 animal. D2 and D3 are of the same regions. D2 shows PV (Cy3) interneurons among the dense plexus of afferent fiber labeling. D3 shows the PV labeling (FITC) in relation to ChAT staining (Cy3). Arrows point to PV-positive interneurons that were consistently lateral to the medial ChAT cell group. Scale bars: All $500 \mu \mathrm{m}$, except C3, $250 \mu \mathrm{m}$.

interneurons, we used an unbiased stereological method, the optical fractionator (West et al., 1991). For this analysis, we used StereoInvestigator (MBF). CBP- and ChAT-positive cells were counted for 5 randomly chosen sections at $200 \times(20 \times$ objective; NA 0.6). A grid size of $150 \mu \mathrm{m}$ and a frame size of $200 \mu \mathrm{m}$ were used to conduct the counts. $\mathrm{CB}$ was counted in laminae 1-3 (from the medial dorsal horn margin to the dorsal peak of the dorsal horn) and laminae 4-6 (from the medial dorsal horn margin to a laterality defined by the dorsal peak of the dorsal horn). These regions corresponded to territories that contained CB-positive cells, as previously reported for the lumbar spinal cord (Anelli and Heckman, 2005). CR and PV were counted in laminae 4-6 (same landmarks as above). ChAT-positive cells were counted in laminae 4-6 (as above) and lamina 7 dorsal (central canal to a laterality defined by the dorsal peak of the dorsal horn). The gray matter borders were drawn at $25 \times$. We chose a sufficient number of sections to analyze so as to minimize variability in cell counts across animals [i.e., Gunderson's CE $(m=1)$; averaged 0.065 for all cases]. We expressed the estimated cell counts for each CBP and ChAT for a $0.2 \mathrm{~mm}$ length of the cord ( 5 sections $\times 40-\mu \mathrm{m}-$ thick section) in each cat as density (cells per square micrometer). We used cell density instead of cell number to allow better comparison across ages and treatments. The mean values for each group is presented as a mean \pm SEM.
Statistical analyses. Standard statistical tests including student's $t$ test and ANOVA were conducted using Microsoft Excel and cluster analyses in JMP 7 (SAS Institute).

\section{Results}

Cervical enlargement interneurons marked with ChAT and calcium-binding proteins

Both ChAT and the CBPs marked both topographically distinct and overlapping interneuron populations. For ChAT and CB, the distributions changed during the CST developmental refinement period. Figure 1 shows representative patterns of ChAT and CBP labeling. These confocal micrographs form the basis of the quantitative localization analyses shown below. The left panel of each part of the figure shows montages obtained by deconvolution confocal microscopy. The right panels contain highermagnification epifluorescence images. Boxes in the left panels show the locations of the higher-magnification images. At PW3 and PW4, ChAT interneurons were consistently present medially in lamina 7, close to the central canal, as previously reported in rat pups (Phelps et al., 1984). There were a few scattered ChAT in- 
terneurons in other locations. Importantly, there was abundant ChAT labeling within the lateral and medial motor pools at these young ages. At PW8 (Fig. 1A1) and older, ChAT interneurons were present both medially in lamina 7 (Fig. $1 A 3$, arrow) and more diffusely throughout laminae 5-7 (Fig. 1 A2).

At PW3 and PW4, CB interneurons were located throughout all laminae outside the motor pools. However, by PW8 there were few labeled cells ventral to laminae 3 and 4 . Figure $1 B 1$ shows CB labeling in a PW3 animal. While the dense superficial band of labeling dominates, scattered cells are present ventrally (Fig. 1B2,B3). A small number of CB-positive neurons in the ventral lamina 7 colocalized PV (Fig. $1 B 3$, inset).

The distributions of CR- (Fig. 1C) (PW3) and PV- (Fig. 1D) (PW3) labeled interneurons were not remarkably different across the ages examined. Laterally located CR interneurons were often large, with polarized dendrites coursing dorsally and ventrally in the gray mater. A small number ( $\sim 1$ per section) of CR-positive neurons in the medial lamina 7 , but lateral to the medial ChAT group, colocalized ChAT (Fig. 1C2, arrows). PV cell body staining tended to be located medially in the gray matter, often in close proximity to the dense axonal PV labeling of largediameter afferent fibers (Fig. 1D1). Figure $1 D 2$ shows PV-positive interneurons among the dense plexus of afferent fiber labeling (Fig. 1 D2, arrow). In medial lamina 7, PV-positive interneurons were consistently present lateral to the medial ChAT cell group (Fig. 1D3; arrows point to $\mathrm{PV}$ interneurons, green; ChAT interneurons are red).

\section{Cervical enlargement interneurons} develop distinctive regional distribution patterns by PW8

Representative examples of regional distribution maps of marked interneurons are shown in Figure 2 for PW4, PW8, and mature cats. The distributions and relative numbers of marked cells appeared to be the same at PW3 as at PW4 (see below). To generate these maps, the color scale was adjusted for each animal. The scale was set so that there was minimal color saturation (i.e., dark red) for each image. Figure 2 presents three key findings. First, ChAT interneurons increase substantially between PW4 and PW8, which is the CST refinement period. Second, CB interneurons decrease substantially between PW4 and PW8. Changes in ChAT and CB density are addressed further below when we describe the results of an unbiased stereological analysis. Third, at all ages, ChAT-, CR-, and PV-positive neurons had distinctive, often highly localized, distributions. These findings show that there is a maturation of the distribution of $\mathrm{CB}$ and ChAT interneurons that parallels
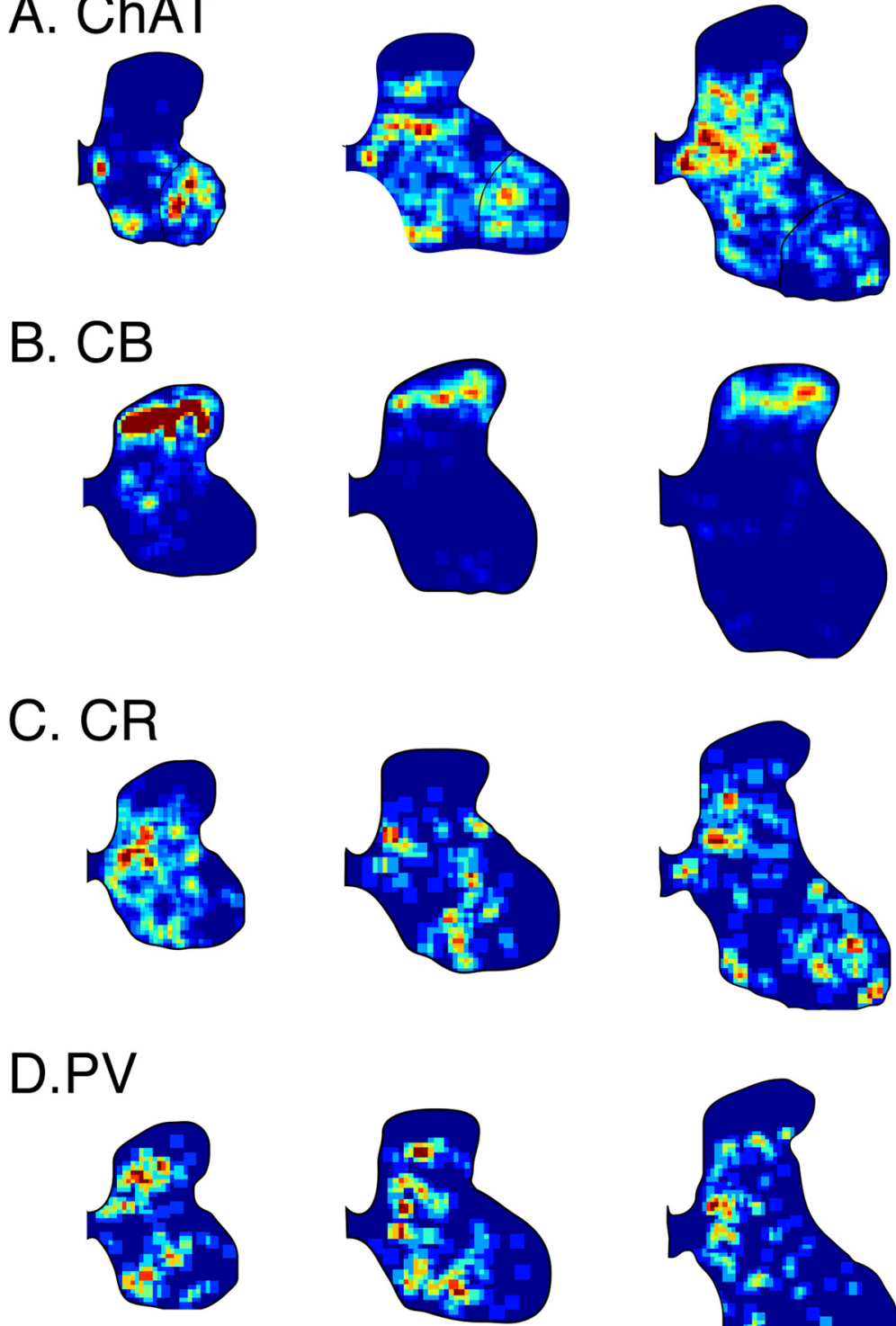

PW8

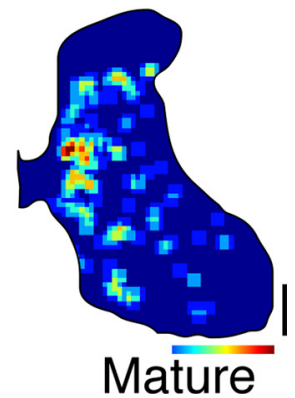

Figure 2. Regional distribution of spinal interneurons at PW4, PW8, and $\geq P W 12$. The locations of labeled interneurons were marked at $100 \times$ magnification in at least two animals per age group. Local density of interneurons (see Materials and Methods) column) from individual animals. Representative examples are shown. $A$, ChAT. Note the increase in ChAT interneurons between PW4 and PW8. B, CB. Note that CB interneuron numbers decreased remarkably between PW4 and PW8. C, CR. D, PV. No agedependent changes were noted for either $(R$ or PV. Scale bar: $500 \mu \mathrm{m}$; range for the color scale (in cells per millimeter): $\boldsymbol{A}, \mathrm{PW} 4$ : 0-33, PW8: 0 -43, adult: 0 -47; B, PW4: 0 -60, PW8: $0-74$, adult: $0-79 ; C$, D, all $0-33$.

the timing of refinement of CST axon terminations and emergence of the M1 motor map (Martin et al., 2009).

\section{Unbiased stereological counts recapitulate changes in regional distribution maps}

To quantify our results of age-dependent changes in interneuron markers, we used the stereological method, optical fractionator, to assess cell numbers in key gray matter regions quantitatively. We combined PW3 and PW4, as a single immature group, and PW8 and $>$ PW12, as a single mature group. This was justified because the results of a hierarchical cluster analysis (supplemental Fig. $1 A$, available at www.jneurosci.org as supplemental material) using a four-age-group model (PW3, PW4, PW8, and 
$\geq$ PW12) as our null hypothesis revealed two principal clusters, immature (blue lines) and mature (green lines). Each cluster had a single cat that was an outlier for its own age group. A second statistical analysis, $K$-means clustering, also produced two principal components, immature and mature, with the same two outliers (supplemental Fig. $1 B$, available at www.jneurosci.org as supplemental material). This provides justification for combining ages and additional support for our hypothesis of interneuronal changes occurring during the CST refinement period.

Figure 3 plots results from the stereological cell counts. For ChAT, we sampled separately lamina 7 dorsal (Fig. 3B), which included the medial ChAT cell group, and laminae 4-6 (Fig. 3A). For both territories, we found a significant increase between the immature (open bar) and mature groups (gray bar; lamina 7, unpaired $t$ test, $p<0.001$, $\mathrm{df}=6$; laminae 4-6, unpaired $t$ test, $p=0.0002, \mathrm{df}=6$ ). Note that the increase in ChAT labeling in lamina 7 reflected substantial increases in numbers of small cells located lateral to the most medial ChAT group, which were present from PW3 (Fig. $2 \mathrm{~A}$ ). We also observed a reduction in $\mathrm{CB}$ labeling between the immature and mature groups, less in laminae $1-3$ (Fig. $2 C$ ) (unpaired $t$ test, $p=0.0139$, $\mathrm{df}=6$ ) than in laminae $4-6$ (Fig. 2D) $(p=0.004, \mathrm{df}=6)$. CR showed a small reduction that was not significant $p=0.34$, $\mathrm{df}=6$ ), as did PV $(p=0.70, \mathrm{df}=6)$. These findings indicate, using an unbiased stereological approach, that there are consistent increases in ChAT-positive interneurons and decreases in CB interneurons before and after the CST refinement period. Thus, as the spinal terminations of the CS system achieve a mature topography between PW5 and PW7, so too do two spinal interneuron classes.

\section{Interneuron clusters are marked by ChAT and CBP staining}

We next defined the locations of clusters of marked interneurons by fitting contours around the densest areas (60\%; see Materials and Methods) of the interneuron regional distribution maps for each cat. This analysis (Fig. 4) combined data across all animals and provides spatial quantification of the locations of interneurons. ChAT neurons were consistently present only in medial lamina 7 at PW3 and PW4 (Fig. 4A, left two columns). This group persisted into maturity. On a given $40 \mu \mathrm{m}$ section, there typically were two to five ChAT-positive cells in this region. By PW8, there was a substantial increase in ChAT labeling throughout the dorsal horn and intermediate zone.

CB-positive interneurons were distributed widely throughout the gray matter outside the motor pools at PW3 and PW4 (Fig. $4 B$, left two columns). At PW8 and mature animals, CB-positive interneurons were localized primarily to the superficial laminae. There were a few CB-positive cells located ventrally (Fig. $2 \mathrm{~B}$ ). One consistent group was located in the ventral portion of lamina 7 , although because of the small number of cells, it fell below the $60 \%$ cutoff. There were no systematic changes in the distribution of clusters of CR and PV interneurons (Fig. 4C,D).

Semischematic summaries of the contour data, split according to whether the animal had an immature CST spinal termination pattern (PW3 and PW4) or a mature pattern (PW8 and mature) are shown in the right two columns of Figure 4 . These summaries clearly show marked differences in ChAT and CB interneurons at immature and mature ages.

From the perspective of the spinal gray matter, superposition of the contours, both in immature and mature animals, shows that there are areas with unique interneuron populations and areas of mixed interneuron groups. The only gray matter zone selective for a single interneuron class in immature animals was the most medial portion of lamina 7 , which contains the medial
A. ChAT lam 4-6

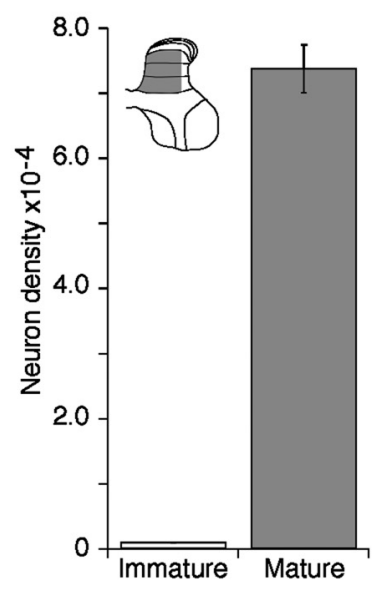

C. CB lam 1-3

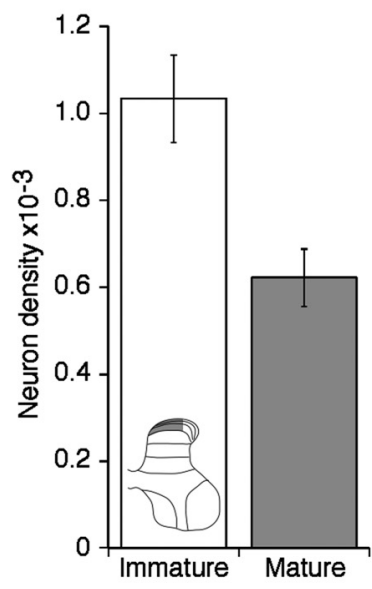

E. CR lam 4-6

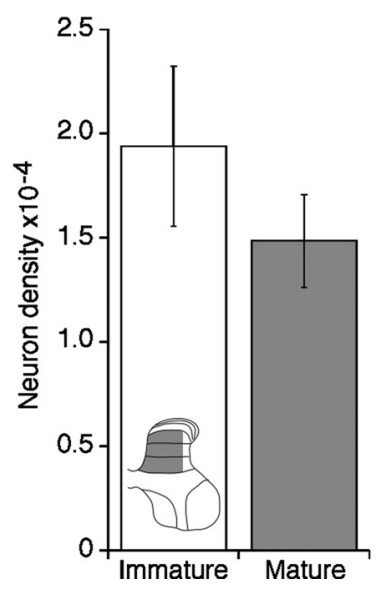

B. ChAT lam 7

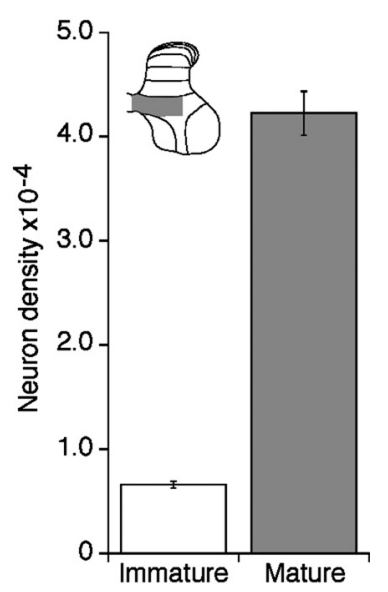

D. CB lam 4-6

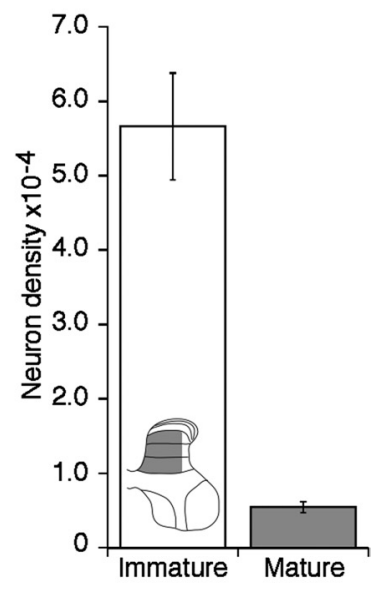

F. PV lam 4-6

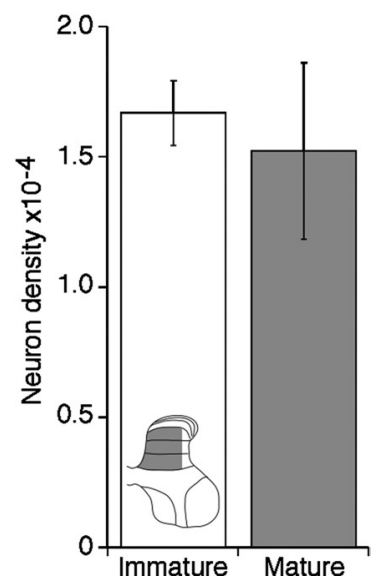

Figure 3. Unbiased measurements of the density of spinal interneurons. The optical fractionator method was used to determine changes in the density of labeled interneurons. The insets in each part show the spinal region measured (gray). Animals were grouped as immature (PW3 and PW4) and mature ( $\geq$ PW8). As discussed in the Results, cluster analysis provided justification for pooling into these two age groups. Cell density was calculated, based on estimated cell counts for each animal in each age group $(5 \times 40 \mu \mathrm{m}$ sections/animal, 4 animals in each group). The mean density for each group and SEM are presented. $A, C$ AT, within laminae 4-6. $B$, ChAT, within lamina 7 dorsal. C, CB, within laminae 1-3.D, CB, within laminae 4-6.E, CR, within laminae 4-6.F, PV, within laminae 4-6. Note that, for the two age groups, there were significant increases in ChAT cell density in both laminae 4 $-6(\boldsymbol{A})$ and lamina $7(\boldsymbol{B})$ and significant decreases in CB density in laminae 1-3 $(\boldsymbol{C}$ and $4-6$ (D). In contrast, $\boldsymbol{E}$ and $\boldsymbol{F}$ show the lack of a significant change in density for CR- and PV-positive neurons in the same animals. lam, Laminae. 


\section{A. ChAT}
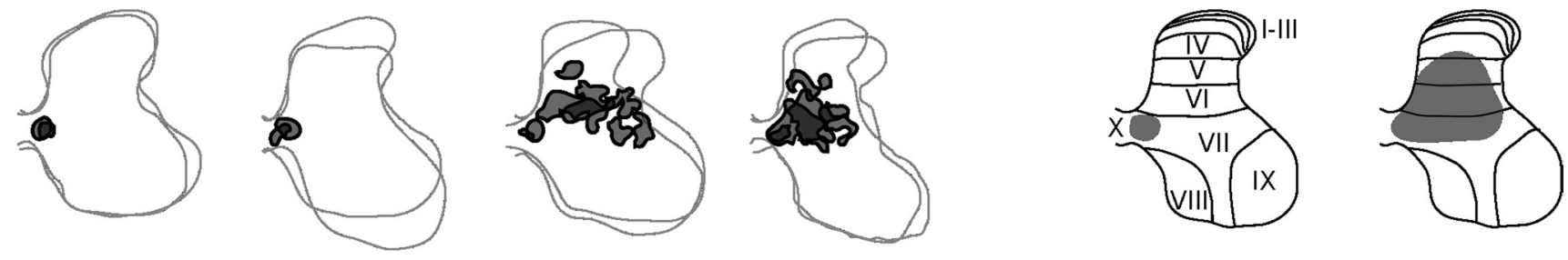

\section{B. $\mathrm{CB}$}
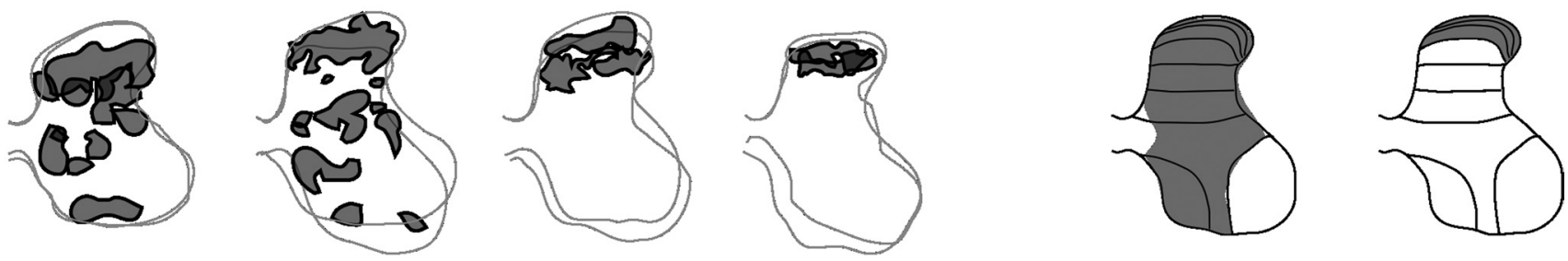

\section{CR}
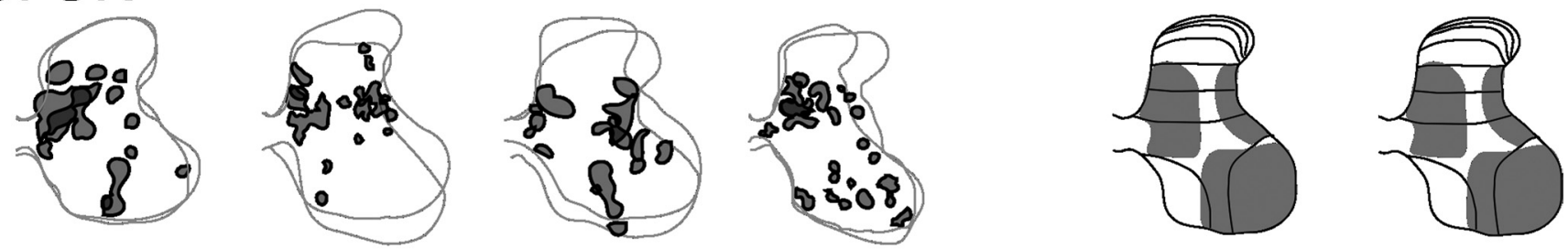

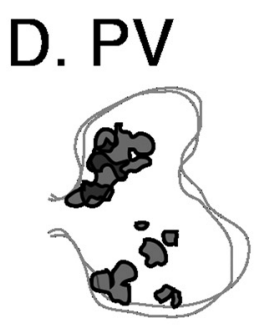

3 wk

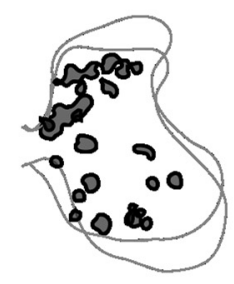

4 wk

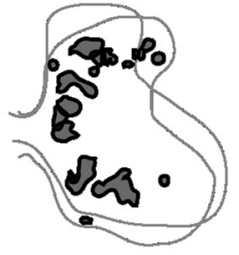

8 wk

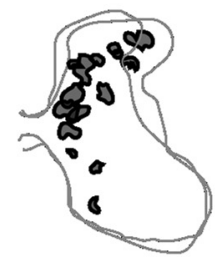

12 wk

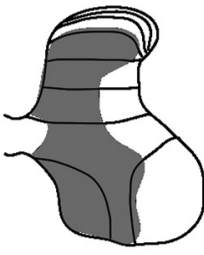

Immature

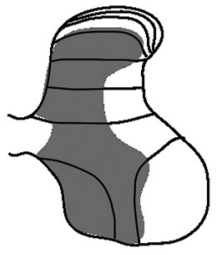

Mature

Figure 4. Interneuron clusters marked by ChAT and CBPs. The left half of the figure shows the locations of clusters (see Materials and Methods for how these were obtained) fitted for all animals (2 per age group). The right two columns are semischematic representations, combining and summarizing the immature (PW3 and PW4) and mature (PW8 and $>$ PW12) groups. The size of all spinal cords was adjusted so that the dorsoventral length of the gray matter was the same. $A$, ChAT neurons were consistently present only in medial lamina 7 at PW3 and PW4 (left two columns). Note the emergence of clusters in the older animals. $\boldsymbol{B}, \mathrm{CB}$ neurons show a clear shift from a diffuse pattern in the immature animals to a focused pattern in laminae $1-3$ in the mature animals. $\boldsymbol{C}$ and $\boldsymbol{D}$ show a diffuse distribution of CR and PV cells, with no apparent change with age. Rexed's laminae are defined in one of the top summary column images.

ChAT cell group. Older than PW8, additional predominantly selective zones emerged: laminae $1-3$, for CB; lamina 4 primarily contained PV interneurons; the middle portion of laminae 5-7 dorsal contained ChAT interneurons primarily; and lateral 5-7 contained predominantly CR cells. The remaining areas had, to varying degrees, combinations of one or more of the markers studied. Supplemental Figure 2 (available at www. jneurosci.org as supplemental material) shows a semischematic summary of the gray matter territories containing the different cell groups. This is discussed further below, in the context of CST termination patterns. Our findings suggest, on the basis of the distributions of interneuron markers, that most zones contain overlapping populations of interneuron classes, and likely overlapping functions. However, there are several regions that have a more selective population of marked interneurons, which may point to a more functionally homogeneous region.

\section{CST terminations target distinctive spinal} interneuron populations

To begin to understand whether the CST targets or avoids connections with particular interneuron populations during development and in maturity, we determined the spatial relationships between the CST projection to the cervical enlargement and the locations of ChAT- and CBP-marked interneurons. We reasoned that CST terminations within the territory of a particular marked interneuron class (e.g., CR in lateral laminae 5-7) (Figs. 2, 4) are likely to make connections with those interneurons since the CST terminations are in the immediate vicinity of the marked interneuron cell bodies. In contrast, terminations that spare the region of a particular labeled interneuron class are less likely to make connections with the interneurons. Although, in the case of the large CR interneuron in lateral lamina 7, their dendritic arbors extend the territory over which contacts can be made. Our contour analysis, which revealed distinctive locations of interneu- 

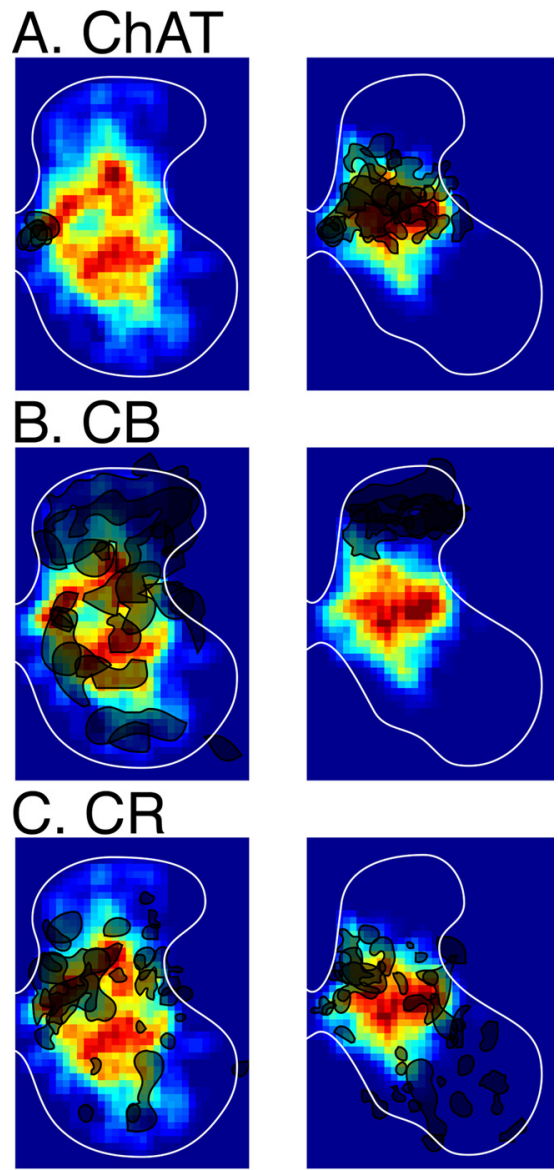

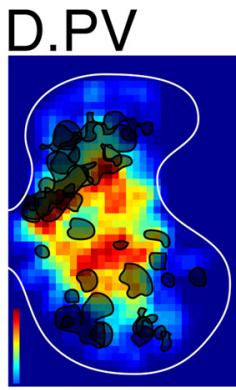

Immature

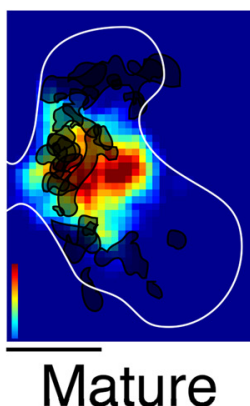

Figure 5. Topographic relationship between composite CST contralateral termination patterns and interneuron cluster overlays. Each composite map was constructed from four animals, two with rostrally placed BDA tracer injections and two with caudally placed injections. $\boldsymbol{A}-\boldsymbol{D}$ overlay interneuron cell clusters on the composite CST distribution maps. Each cell cluster from each animal is shown as a black outline and $50 \%$ transparent fill. The greater the overlap between animals, the darker the fill. As in the analysis for Figure 4, to facilitate spatial comparisons the dorsoventral length of the gray matter was adjusted to be the same in all animals. $\boldsymbol{A}$, ChAT. $\boldsymbol{B}$, Calbindin. C, Calretinin. D, Parvalbumin. Scale bars: $1 \mathrm{~mm}$; range for color scale: length, $0-800 \mu \mathrm{m}$ axon length.

rons identified by ChAT and CBPs, provides the basis for this analysis. We conducted two separate analyses one based on composite CST axon labeling (see Materials and Methods) and one based on the distributions of CST axons and varicosities (i.e., boutons; see Materials and Methods) and interneurons in individual animals.

Figure 5 overlays the distributions of ChAT- and CBP-labeled interneurons (black outlines and semitransparent fill; darker fill indicates more overlap between animals) on composite CST axon maps. As predicted from earlier studies (Theriault and Tatton,
1989; Li and Martin, 2000), CST labeling in immature animals covers most of the gray matter, including superficial laminae and the motor pools (Fig. 5, left). Labeling at this age is densest contralaterally, but also present ipsilaterally (Theriault and Tatton, 1989; Alisky et al., 1992; Martin et al., 1999). Moreover, densest labeling was within the deep dorsal horn and intermediate zone. In mature animals, labeling was more restricted dorsoventrally (Fig. 5, right).

For ChAT, the immature CST projections terminate predominantly within the future location of the more lateral placed interneurons. The composite map in Figure $5 \mathrm{~A}$ (left) shows labeling located lateral to the territory of the medial ChAT cells in lamina 7. As discussed below, projections to this region were sparse in PW3 and PW4 animals, but were denser by 8 weeks. In the mature animal (Fig. 5A, right), there was a remarkable correspondence between the region of densest contralateral CST labeling and the territory occupied by ChAT cells. For CB, the sparse CST projection to the superficial dorsal horn in immature animals overlaps the high density interneuronal population (Fig. 5B, left). Postnatal withdrawal of the superficial CST projections (Theriault and Tatton, 1989; Alisky et al., 1992; Li and Martin, 2001) occurs in parallel with the reduction in the overall level of $\mathrm{CB}$ expression in that region (Fig. 3). Whereas the CST projection is maintained in the deep dorsal horn and intermediate zone, there is a corresponding large-scale reduction in $\mathrm{CB}$ expression, so much so in laminae $4-8$ that groups dropped out (Fig. $5 B$, right). Not surprisingly, for the immature CST there was general overlap with CR- and PV-positive cells, since these interneuronal markers are diffuse (Fig. 5C,D). After refinement of CST terminations, the most dorsal and most ventral PV and CR cells remained outside the CST termination territory. Importantly, the regions with densest CST labeling, the medial portions of laminae 5-7 dorsal, target the area containing a high density of CR- and PV-positive neurons. The findings that ChAT immunoreactivity increases and $\mathrm{CB}$ immunoreactivity decreased in temporal and spatial association with CST terminations raises the possibility that the CST plays a role in these directing these changes (see below).

We next compared focal densities of CST labeling with interneuronal labeling in the same animal (Fig. 6; axon labeling, left panels; varicosity labeling, right panels). We focused primarily on the correspondence between the medial ChAT neurons, which formed a readily identifiable population, and CST terminations. This analysis supports our observation that the medial ChAT neurons in lamina 7 were not targeted by the immature CST projection. Often, there were conspicuous gaps in the distribution of labeling suggesting that the projection avoided this region (Fig. 6, PW4). Rather, CST terminations in the younger animals were consistently located more lateral, but still within the medial portions of laminae 5-7 dorsal. This region contained cells, singly labeled for all four markers (Fig. 4, right columns; supplemental Fig. 2, available at www.jneurosci.org as supplemental material). There was a focus of dense varicosities in the medial portion of laminae 5-6 (Fig. 6B, upper arrow) that was distinct from more ventral projections (Fig. $6 \mathrm{~B}$, lower arrow). In more mature animals, we saw more medial lamina 7 labeling, close to the central canal, that was localized within the medial ChAT-positive cells. In the PW8 animal shown, though the densest labeling was in medial laminae 5 and 6 , a lower density region of varicosities extends to the medial ChAT cells (Fig. 6C). Tracer injection in the PW12 animal (Fig. 6D) labeled several clusters within lamina 7, including the medial ChAT cell group (white contour) and the lateral CR group (arrows).

Two different tracers were used in another PW12 animal (Fig. 
6E; more caudal M1 injections, E1, E2; more rostral M1 injections, E3, E4). Whereas the distribution of labeling from the adjoining regions were similar-both targeted laminae 5-7-closer examination revealed important differences that only can be interpreted in the context of the locations of labeled interneuron groups: the more rostral injection targeted the medial ChAT group (Fig. 6E3,E4), whereas the more caudal one did not (Fig. $6 E 1, E 2)$. The lateral CR-positive region received a projection from one (Fig. 6E3,E4) but not the other (Fig. 6E1,E2) M1 region. Overall, the termination patterns from the two adjoining areas were remarkably interdigitated (Fig. 6E1,E3), suggesting a high degree of connectional specificity between M1 and spinal motor circuits. Our findings suggest that the CST targets distinctive populations of spinal interneurons at different times during development.

Activity-dependent changes in CB- and ChAT-positive interneurons

The findings presented above show that $\mathrm{CB}$ expression decreases, and ChAT increases, during the period when CST axon terminations are refined. This raises the possibility that these changes depend on a functioning CST input. To test this idea, we inactivated M1 unilaterally during the CST refinement period, PW5-PW7, and examined the distributions of CBPs and ChAT contralateral and ipsilateral to inactivation. Three cats were 8 weeks old and two were 12 weeks old at the time of perfusion. We combined data from these two age groups because no systematic differences were observed. This inactivation produces, without further activity-based intervention, a permanent shift in the distribution of inactivated CST terminations, mostly dorsal to lamina 4 (Friel and Martin, 2005). Inactivation also produces a permanent behavioral impairment, in which cats show movement endpoint biases during reaching (overreaching) and visually guided stepping (overstepping) and grasping impairments (Martin et al., 2000; Friel et al., 2007).

Figure $7 A$ shows the regional density of $C B$-positive interneurons in a representative animal. There were noticeably more CBpositive interneurons on the inactive (contralateral to inactivation) than the active (ipsilateral to inactivation) side in laminae $1-3$. We counted the number of CB-positive interneurons using the optical fractionator, as above, and found more than a doubling of neuron density on the inactive than active side (Fig. 7C). This difference was significant (paired $t$ test, $p<0.01$ ). No sideto-side differences were observed in an animal that received unilateral saline infusion during the same period (contralateral to infusion: $6.78 \times 10^{-4}$ cells/ $\mu \mathrm{m}^{2}$; ipsilateral to infusion: $6.83 \times$ $10^{-4}$ cells $\left./ \mu \mathrm{m}^{2}\right)$. Relative to the active side, it was as if unilateral inactivation prevented the reduction in CB expression in the superficial laminae that normally occurs between PW5 and PW7 (Fig. $2 B$ ).
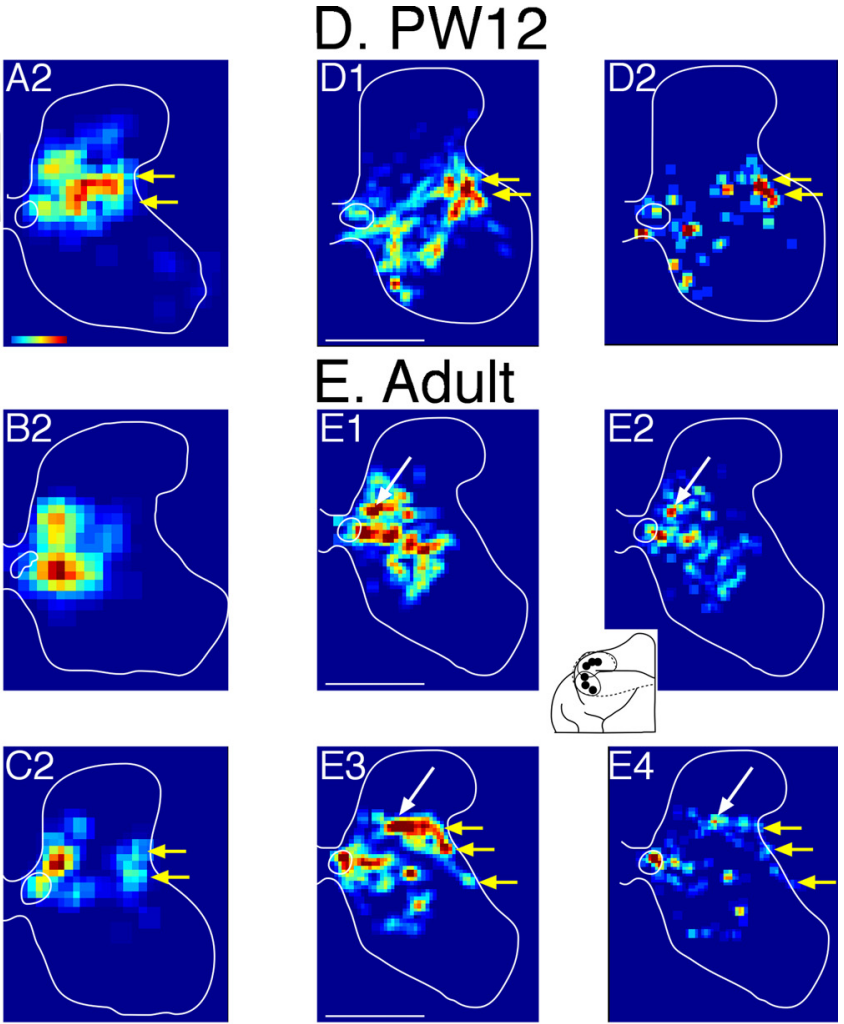

Varicosities

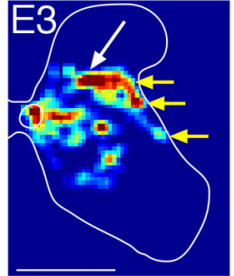

Axons

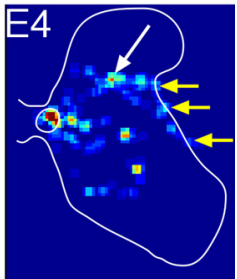

Varicosities
Figure 6. Targeting of CST projections to particular contralateral spinal subregions. Each panel shows the regional distribution ChAT cell group at PW3 and PW4 C. PW8. CST labeling now extends into the region of the medial ChAT group Yellow arrows point

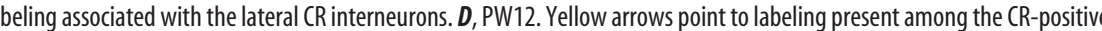
color scale range: left panels, axon length: $0-400 \mathrm{~mm}$ of axon length; right panels, varicosities (number per millimeter): $\boldsymbol{A}-\boldsymbol{C}$, $0-120 ; D, 0-60 ; E 2,0-115 ; E 4,0-80$.

Surprisingly, CB interneuron density on the inactive side was not significantly different from age-matched controls (means: inactive, $5.47 \times 10^{-4} \pm 2.85 \times 10^{-5}$; controls, $6.21 \times 10^{-4} \pm$ $6.55 \times 10^{-5}$; unpaired $t$ test, $\left.p=0.2951, \mathrm{df}=7\right)$; CB density on the active side $\left(2.33 \times 10^{-4} \pm 3.09 \times 10^{-5}\right)$ was significantly less than the inactive side (paired $t$ test, $p<0.0001$, df $=8$ ). However, the relative difference in $\mathrm{CB}$ interneuron counts between the inactive and active sides (i.e., high and low numbers, respectively) was the same as the relative difference for immature (i.e., PW3 and PW4) and mature (i.e., PW8 and adult) cats.

An asymmetry in ChAT-positive interneurons also was produced by unilateral inactivation. Figure $7 B$ shows the regional density of ChAT-positive interneurons in a representative animal. The inactive side (contralateral to inactivation) contained significantly fewer ChAT-positive interneurons than the active side, as shown in Figure $7 D$ (paired $t$ test, $p<0.01$ ). Side-to-side asymmetry was not present in the animal subjected to saline infusion (contralateral to infusion: $3.45 \times 10^{-4}$; ipsilateral to infusion: $\left.3.26 \times 10^{-4}\right)$. Thus, as with $\mathrm{CB}$, inactivation produced less mature (i.e., lower) regional density of ChAT-positive interneurons in the contralateral spinal gray matter.

ChAT interneuron density was significantly less on the inac- 

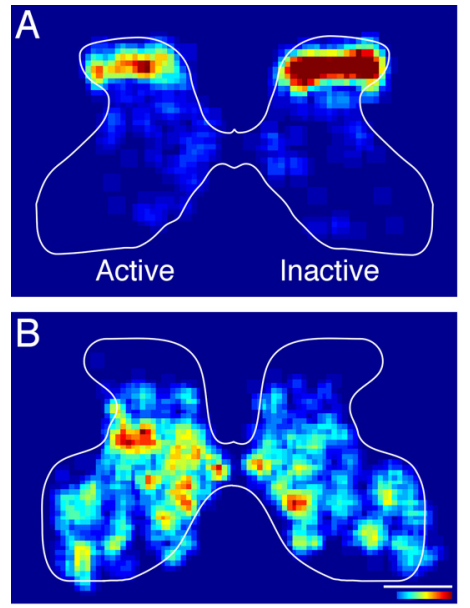
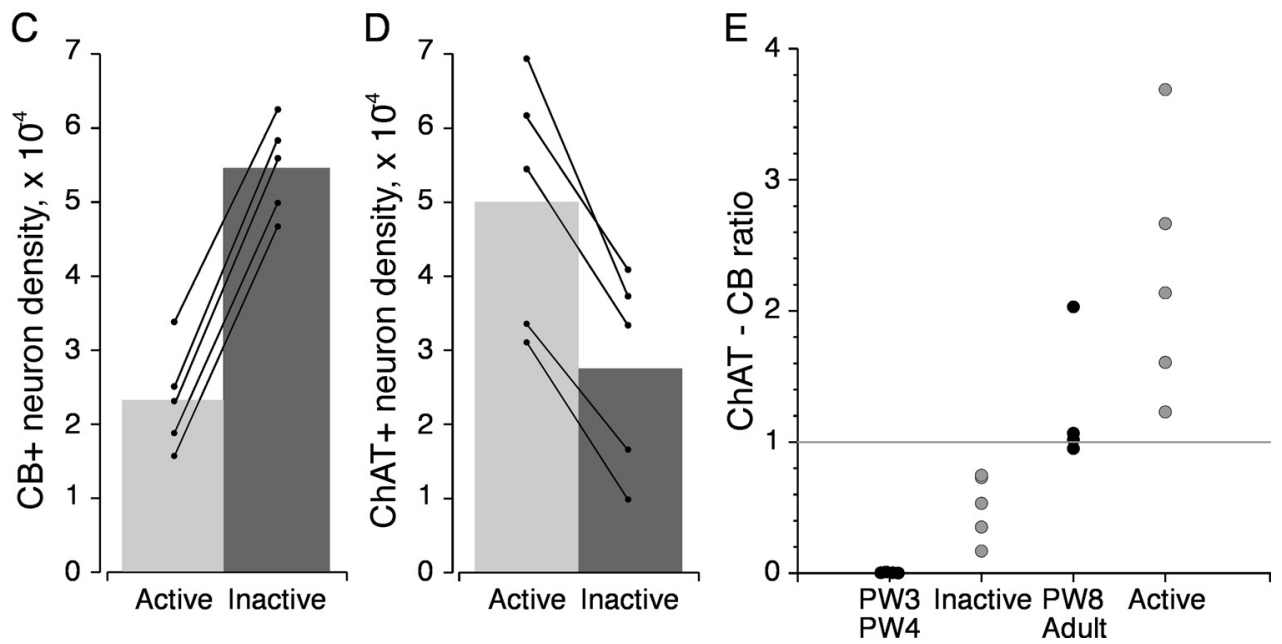

Figure 7. Development of $\mathrm{CB}$ and $\mathrm{ChAT}$ interneurons depends on $\mathrm{M} 1$ activity. $A$, Representative regional distribution of $\mathrm{CB}$ interneurons in the cervical spinal cord contralateral (right) and ipsilateral (left) to $\mathrm{M} 1$ inactivation. Cell density is represented as a color scale. $B$, Similar to $A$, but for ChAT. C, Unbiased measurement of CB interneuron density, using the optical fractionator method. Note that there is more than a doubling of neuron density on the inactive over the active side. The bar graph plots mean. Data from individual animals are shown. $\boldsymbol{D}$, As in $\boldsymbol{C}$, but for $C h A T$ interneuron density. $\boldsymbol{E}$, Ratio of ChAT to CB, calculated for each animal. Scale bar: (in $\boldsymbol{B}) \boldsymbol{A}, \boldsymbol{B}, 1 \mathrm{~mm}$. Color scale range: in $(\boldsymbol{B}) \boldsymbol{A}, 0-75$ cells $/ \mathrm{mm} ; \boldsymbol{B}, 0-30$ cells $/ \mathrm{mm}$.

tive side than in age-matched controls (means: inactive, $2.76 \times$ $10^{-4} \pm 6.09 \times 10^{-5}$; controls, $7.37 \times 10^{-4} \pm 4.82 \times 10^{-5}$, unpaired $t$ test $p<0.01)$. ChAT neuron density was significantly higher on the active side $\left(5.01 \times 10^{-4} \pm 7.61 \times 10^{-5}\right.$; paired $t$ test, $p=0.05$, $\mathrm{df}=8$ ). While this is different from $\mathrm{CB}$, where inactive and age-matched controls were the same, ChAT also showed the same relative difference with respect to younger animals. ChAT interneuron counts were lower on the inactive sides, which is the same as immature cats, and higher on the active side, as in mature cats.

In contrast to affecting $\mathrm{CB}$ and ChAT interneuron development, CS system inactivation had no effect on PV and CR interneuron development (PV, PW3 and PW4: $1.67 \times 10^{-4}$, controls: $1.54 \times 10^{-4}$, paired $t$ test, $p=0.57, \mathrm{CR}, \mathrm{PW} 3$ and PW4: $1.94 \times$ $10^{-4}$, controls: $1.49 \times 10^{-4}$, paired $t$ test, $\left.p=0.44\right)$. These findings support our hypothesis that CS system activity is necessary for the postnatal changes in ChAT and CB.

On the inactive side, the density of CB-positive interneurons was high and the density of ChAT interneurons was low. This is the general pattern of $\mathrm{CB}$ and ChAT in immature animals. In contrast, on the active side the density of CB-positive interneurons was low and the density of ChAT interneurons was high. This pattern was like that in mature animals. Our hypothesis is that the ChAT-CB ratio on the inactive side, rather than the absolute numbers of cells, will be similar to that in immature animals and that the ratio on the active side, will be similar to that in mature animals. We next compared the ratio of ChAT-positive to CB-positive interneuron density for animals subjected to unilateral inactivation, with that in normally developing animals (Fig. 7E). As predicted, PW3 and PW4 animals have an immature CST topography and they also have a ChAT-CB ratio close to zero. PW8 and older animals have a CST with a mature termination pattern and they have a ChAT-CB ratio of one and greater (Fig. 7E, black dots). After unilateral inactivation, the inactive side (gray dots) had ratios that were less than one, similar to the immature animals, and the active side (gray dots), greater than one, as with the mature animals. These results support our hypothesis that interneurons within the CST spinal target zone contralateral to CS system inactivation have a more immature ChAT-CB pattern, than the active side.

\section{Discussion}

The CS system becomes functionally important as a developing motor pathway by PW8 in the kitten, when the spinal terminations achieve their mature topographic distribution, connection strength increases, and the M1 motor map begins to function (Martin et al., 2009). This is also when kittens show stable motor behaviors and engage in play with objects (Villablanca and Olmstead, 1979; Martin and Bateson, 1985). The observed changes in spinal interneuron marker expression likely reflect functional modifications rather than cell number changes (i.e., cell birth or death). We found changes in ChAT and CB expression in spinal interneurons as the CST matures and within the laminae where CST termination density changes. This association suggests that the developing CS system plays a role in directing interneuron maturation in a temporospatially specific way. Consistent with this view is that development of the mature ChAT- and CBPinterneuron distributions depends on CS system activity.

Rather than project indiscriminately to wide regions of the spinal gray matter (Theriault and Tatton, 1989; Alisky et al., 1992), we found that developing CST axon terminations show an early specificity in relation to particular interneuron groups that is subsequently refined, with age, activity, and experience (Martin and Lee, 1999; Martin et al., 1999, 2004). Connections are eliminated-including within the CB-containing superficial laminae (Theriault and Tatton, 1989; Li and Martin, 2000)—whereas others are late developing, such as the most medial ChAT interneurons in lamina 7. CST projections to medial laminae 5 and 6, which contain a diversity of interneuron types, are present early in development and largely maintained. This is a target of the CST in diverse species (Porter and Lemon, 1993). This region contains cerebellar projection neurons (Verburgh et al., 1989) and receives inputs from large-diameter fibers (Martin and Chakrabarty, 2008). Our marking methods focused on CST-cell body colocalization, since most interneurons had local and laminar specific dendritic arbors and axosomatic synapses are apt to be strong. However, there may be important CST inputs onto distal dendrites of neurons whose cell bodies are outside the field of dense CST terminations. 


\section{What are the interneuronal targets of the developing CST?}

We found clear clustering of marked interneurons and consistent associations between particular interneuron clusters and foci of CST terminations. Clear differences were noted for ChAT and CB. Immature CST terminations largely spare medial lamina 7 ChAT cells, and terminate in fields where ChAT expression is initially low but will increase substantially over the next few weeks. The medial lamina 7 ChAT neurons are likely premotor interneurons making C-bouton contacts (Miles et al., 2007) with muscarinic receptors on motoneurons (Hellström et al., 2003; Wilson et al., 2004). Muscarinic agonists increase a motoneuron's response to injected current (Miles et al., 2007). Cholinergic neurons in medial lamina 7 are active during fictive locomotion (Huang et al., 2000). The finding that CST axons may avoid this premotor target early in development suggests that at early ages the CST lacks oligosynaptic access to motoneurons via this connection, limiting expression of CST motor functions. The densest territory of CST axons is where ChAT interneurons later emerge. Low levels of ChAT interneuron expression likely reflect a lack of functional cholinergic connections with more ventral postsynaptic targets that increase expression with development of more effective connections. Here too, the CS system is denied access to motoneurons early in development.

$\mathrm{CB}$ is typically associated with inhibition (Kubota and Jones, 1993; Schwaller et al., 2002). CB dominated the superficial laminae, where the developing CST has projections (Theriault and Tatton, 1989; Li and Martin, 2000). As CST terminations are lost from these laminae by PW8, CB expression decreases. By the ages tested, there were no major changes in PV and CR during the CST refinement period. Prior reports showing an increase in PV correlated this change with development of large-diameter afferent input to the cord (for review, see Clowry, 2007). However, this has occurred by PW3 in the cat (Martin and Chakrabarty, 2008).

\section{Model for developmental changes in ChAT and CB}

Our hypothesis is that the CST provides trophic support to its targets, such as by activity-dependent release of BDNF or NT3 (Giehl, 2001). During normal development, this support for the CB neurons in laminae 1-3 would be withdrawn as CST terminations are eliminated. For ChAT, trophic support could increase as the CST strengthens its connections during development (Martin et al., 2009). The raised CST drive increases ChAT expression, since neurotransmitter phenotype can be activity dependent (Spitzer and Borodinsky, 2008). We further propose that the ChAT-CB ratio is an index of the balance between excitation (ChAT) and inhibition (CB). During normal development, immature animals have a ChAT-CB ratio $<1$, resulting in net inhibition. For ChAT and CB cells that are CST targets, this would limit transmission from $\mathrm{M} 1$ to muscle. Mature animals have a ChAT-CB ratio $>1$, or net excitation, permitting CS activation of muscle. Changes in CB development supplement those discussed above for ChAT and bolster our hypothesis that the immature spinal gray matter is inhibitory to CST signaling.

During muscimol infusion, CST activation of the contralateral spinal gray matter is low, limiting activity-dependent maturational changes in CBP and ChAT. In addition, contralateral CST terminations are shifted to more superficial laminae (Friel and Martin, 2005), which could further contribute to the observed changes: superficial CB neuron density is maintained and there are fewer ChAT-positive neurons in the deeper laminae.

While the relative changes in $\mathrm{ChAT}$ and $\mathrm{CB}$ neuron density after inactivation resembled immature animals (i.e., ChAT-CB ratio $<1$ ), CB was not as high and ChAT was higher than in immature controls. CB was not as high because (1) CB expression was diminishing when the inactivation began at PW5, and (2) CST terminations after inactivation appeared sparser than in immature controls ( $\mathrm{Li}$ and Martin, 2000), providing less trophic support. Together, these factors help explain why $\mathrm{CB}$ cell density was at the normal mature, rather than immature, level. The number of ChAT cells was higher than in immature animals because their numbers were rising at the onset of inactivation and the aberrant ipsilateral CST terminations could provide support.

Our trophic support model also helps explain why the numbers of $\mathrm{CB}$ and $\mathrm{ChAT}$ neurons on the active side of the cord are lower than mature controls. Fewer numbers could reflect a reduced capacity for the active CST to provide trophic support to its expanded, now bilateral, target field, as suggested by homeostatic models (Turrigiano and Nelson, 2004). This also predicts that the bilateral terminations would be vulnerable to withdrawal later in development, which we recently reported (Friel and Martin, 2007), because of limited trophic support. Rather than maintain the normal interneuron numbers on the active side, the ChAT-CB ratio was like mature controls $(>1)$. Normalization of the ratio could reflect a homeostatic process, as seen in other systems to regulate overall levels of excitability (Turrigiano and Nelson, 2004).

\section{Multiple functional corticospinal tracts}

Historically, the CST has been considered a unitary structure for feedforward motoneuron control, but this view is undergoing revision (Lemon, 2008). The diversity of CST connections in the spinal cord calls attention to a functional diversity. Different M1 regions can contact selective interneuronal populations. For example, rostral M1 projections are ventrally biased, compared with caudal M1, and the only subregion with connectivity to C3-C4 propriospinal neurons (Martin, 1996; Alstermark and Ohlson, 2000). Our findings suggest an even more subtle organization, in which neighboring territories have interdigitated terminations. A human degeneration study (Schoen, 1964) showed a remarkably punctate projection to medial laminae 5 and 6 in the cervical enlargement. This is the region in which we find diverse CBP/ChAT labeling. That same study showed terminal degeneration in the central region of dorsal lamina 7 , which is where we observed increased ChAT expression during development that was muted with M1 inactivation.

\section{Development of a M1 functional motor map}

Before CST refinement, M1 microstimulation fails to evoke movement (Bruce and Tatton, 1980; Chakrabarty and Martin, 2000). At about PW7-PW8, relatively high currents evoke proximal joint movements from a few cortical locations (Chakrabarty and Martin, 2000). By PW12 all forelimb joints are represented at low current thresholds. The presence of an M1 motor map later in development and maturity correlates with skilled limb control (Monfils et al., 2005; Chakrabarty et al., 2009). Our finding that the CST targets cholinergic interneuron populations with strong ChAT expression after the CST refinement period provides an important spinal substrate for map development. With connections to effective spinal ChAT networks, M1 could connect functionally with motoneurons. Furthermore, M1 activity blockade between PW5 and PW7 produces aberrant dorsal CST projections (Friel and Martin, 2005) that are outside the field of most ChAT neurons, impairing M1 motor map development (Chakrabarty et al., 2009) and limb control (Martin et al., 2000; Friel et al., 2007). Interventions that return CST terminations to more ventral laminae, restores the M1 motor map and limb control (Friel and Martin, 2007; Salimi et al., 2008; Chakrabarty et al., 2009). Our findings provide strong support for 
the importance of development of the spinal connections of the CST to particular interneuronal populations as a key event in shaping of the M1 motor map and expression of CS system motor functions.

\section{References}

Albuquerque C, Lee CJ, Jackson AC, MacDermott AB (1999) Subpopulations of GABAergic and non-GABAergic rat dorsal horn neurons express Ca2 +-permeable AMPA receptors. Eur J Neurosci 11:2758-2766.

Alisky JM, Swink TD, Tolbert DL (1992) The postnatal spatial and temporal development of corticospinal projections in cats. Exp Brain Res $88: 265-276$

Alstermark B, Ohlson S (2000) Origin of corticospinal neurones evoking monosynaptic excitation in C3-C4 propriospinal neurones in the cat. Neurosci Res 38:249-256.

Anelli R, Heckman CJ (2005) The calcium binding proteins calbindin, parvalbumin, and calretinin have specific patterns of expression in the gray matter of cat spinal cord. J Neurocytol 34:369-385.

Armand J, Holstege G, Kuypers HG (1985) Differential corticospinal projections in the cat. An autoradiographic tracing study. Brain Res 343:351-355.

Armand J, Olivier E, Edgley SA, Lemon RN (1997) Postnatal development of corticospinal projections from motor cortex to the cervical enlargement in the macaque monkey. J Neurosci 17:251-266.

Bruce IC, Tatton WG (1980) Synchronous development of motor cortical output to different muscles in the kitten. Exp Brain Res 40:349-353.

Brus-Ramer M, Carmel JB, Chakrabarty S, Martin JH (2007) Electrical stimulation of spared corticospinal axons augments connections with ipsilateral spinal motor circuits after injury. J Neurosci 27:13793-13801.

Celio MR, Baier W, Schärer L, de Viragh PA, Gerday C (1988) Monoclonal antibodies directed against the calcium binding protein parvalbumin. Cell Calcium 9:81-86.

Chakrabarty S, Martin JH (2000) Postnatal development of the motor representation in primary motor cortex. J Neurophysiol 84:2582-2594.

Chakrabarty S, Friel KM, Martin JH (2009) Activity-dependent plasticity improves M1 motor representation and corticospinal tract connectivity. J Neurophysiol 101:1283-1293.

Clowry GJ (2007) The dependence of spinal cord development on corticospinal input and its significance in understanding and treating spastic cerebral palsy. Neurosci Biobehav Rev 31:1114-1124.

Clowry GJ, Arnott GA, Clement-Jones M, Fallah Z, Gould S, Wright C (2000) Changing pattern of expression of parvalbumin immunoreactivity during human fetal spinal cord development. J Comp Neurol 423:727-735.

Clowry GJ, Davies BM, Upile NS, Gibson CL, Bradley PM (2004) Spinal cord plasticity in response to unilateral inhibition of the rat motor cortex during development: changes to gene expression, muscle afferents and the ipsilateral corticospinal projection. Eur J Neurosci 20:2555-2566.

Friel KM, Martin JH (2005) Role of sensory-motor cortex activity in postnatal development of corticospinal axon terminals in the cat. J Comp Neurol 485:43-56.

Friel KM, Martin JH (2007) Bilateral activity-dependent interactions in the developing corticospinal system. J Neurosci 27:11083-11090.

Friel KM, Drew T, Martin JH (2007) Differential activity-dependent development of corticospinal control of movement and final limb position during visually-guided locomotion. J Neurophysiol 97:3396-3406.

Gibson CL, Arnott GA, Clowry GJ (2000) Plasticity in the rat spinal cord seen in response to lesions to the motor cortex during development but not to lesions in maturity. Exp Neurol 166:422-434.

Giehl KM (2001) Trophic dependencies of rodent corticospinal neurons. Rev Neurosci 12:79-94.

Gonzalez-Forero D, Pastor AM, Geiman EJ, Benítez-Temiño B, Alvarez FJ (2005) Regulation of gephyrin cluster size and inhibitory synaptic currents on Renshaw cells by motor axon excitatory inputs. J Neurosci 25:417-429.

Hellström J, Oliveira AL, Meister B, Cullheim S (2003) Large cholinergic nerve terminals on subsets of motoneurons and their relation to muscarinic receptor type 2. J Comp Neurol 460:476-486.

Huang A, Noga BR, Carr PA, Fedirchuk B, Jordan LM (2000) Spinal cholinergic neurons activated during locomotion: localization and electrophysiological characterization. J Neurophysiol 83:3537-3547.

Kubota Y, Jones EG (1993) Co-localization of two calcium binding proteins in GABA cells of rat piriform cortex. Brain Res 600:339-344.
Lemon RN (2008) Descending pathways in motor control. Annu Rev Neurosci 31:195-218.

Li Q, Martin JH (2000) Postnatal development of differential projections from the caudal and rostral motor cortex subregions. Exp Brain Res 134:187-198.

Li Q, Martin JH (2001) Postnatal development of corticospinal axon terminal morphology in the cat. J Comp Neurol 435:127-141.

Martin J, Chakrabarty S (2008) Early postnatal co-development of corticospinal and proprioceptive circuits in the spinal cord. Soc Neurosci Abstr 34:29.12.

Martin J, Friel K, Salimi I, Chakrabarty S (2009) Corticospinal development. In: Encyclopedia of neuroscience (Squire L, ed), pp 203-214. Oxford: Academic.

Martin JH (1996) Differential spinal projections from the forelimb areas of the rostral and caudal subregions of primary motor cortex in the cat. Exp Brain Res 108:191-205.

Martin JH, Lee SJ (1999) Activity-dependent competition between developing corticospinal terminations. Neuroreport 10:2277-2282.

Martin JH, Kably B, Hacking A (1999) Activity-dependent development of cortical axon terminations in the spinal cord and brain stem. Exp Brain Res 125:184-199.

Martin JH, Donarummo L, Hacking A (2000) Impairments in prehension produced by early postnatal sensorimotor cortex activity blockade. J Neurophysiol 83:895-906.

Martin JH, Choy M, Pullman S, Meng Z (2004) Corticospinal development depends on experience. J Neurosci 24:2122-2132.

Martin P, Bateson P (1985) The ontogeny of locomotor play behavior in the domestic cat. Anim Behav 33:502-510.

Meng Z, Li Q, Martin JH (2004) The transition from development to motor control function in the corticospinal system. J Neurosci 24:605-614.

Miles GB, Hartley R, Todd AJ, Brownstone RM (2007) Spinal cholinergic interneurons regulate the excitability of motoneurons during locomotion. Proc Natl Acad Sci U S A 104:2448-2453.

Monfils MH, Plautz EJ, Kleim JA (2005) In search of the motor engram: motor map plasticity as a mechanism for encoding motor experience. Neuroscientist 11:471-483.

Phelps PE, Barber RP, Houser CR, Crawford GD, Salvaterra PM, Vaughn JE (1984) Postnatal development of neurons containing choline acetyltransferase in rat spinal cord: an immunocytochemical study. J Comp Neurol 229:347-361.

Porter R, Lemon R (1993) Corticospinal function and voluntary movement. Oxford: Oxford Science.

Rexed B (1954) The cytoarchitectonic organization of the spinal cord in the cat. J Comp Neurol 100:297-379.

Salimi I, Friel KM, Martin JH (2008) Pyramidal tract stimulation restores normal corticospinal tract connections and visuomotor skill after early postnatal motor cortex activity blockade. J Neurosci 28:7426-7434.

Schoen JH (1964) Comparative aspects of the descending fibre systems in the spinal cord. Prog Brain Res 11:203-222.

Schwaller B, Meyer M, Schiffmann S (2002) 'New' functions for 'old' proteins: the role of the calcium-binding proteins calbindin D-28k, calretinin and parvalbumin, in cerebellar physiology. Studies with knockout mice. Cerebellum 1:241-258.

Spitzer NC, Borodinsky LN (2008) Implications of activity-dependent neurotransmitter-receptor matching. Philos Trans R Soc Lond B Biol Sci 363:1393-1399.

Theriault E, Tatton WG (1989) Postnatal redistribution of pericruciate motor cortical projections within the kitten spinal cord. Brain Res Dev Brain Res 45:219-237.

Turrigiano GG, Nelson SB (2004) Homeostatic plasticity in the developing nervous system. Nat Rev Neurosci 5:97-107.

Verburgh CA, Kuypers HG, Voogd J, Stevens HP (1989) Spinocerebellar neurons and propriospinal neurons in the cervical spinal cord: a fluorescent double-labeling study in the rat and the cat. Exp Brain Res 75:73-82.

Villablanca JR, Olmstead CE (1979) Neurological development of kittens. Dev Psychobiol 12:101-127.

West MJ, Slomianka L, Gundersen HJ (1991) Unbiased stereological estimation of the total number of neurons in the subdivisions of the rat hippocampus using the optical fractionator. Anat Rec 231:482-497.

Wilson JM, Rempel J, Brownstone RM (2004) Postnatal development of cholinergic synapses on mouse spinal motoneurons. J Comp Neurol 474: $13-23$. 